\title{
Observation of surface charge screening and Fermi level pinning on a synthetic, boron-doped diamond
}

\author{
Shenda M. Baker, a) George R. Rossman, ${ }^{\text {b) }}$ and John D. Baldeschwieler \\ Division of Chemistry and Chemical Engineering, California Institute of Technology, Pasadena, \\ California 91125
}

(Received 8 December 1992; accepted for publication 21 May 1993)

\begin{abstract}
Spectroscopic current-voltage $(I-V)$ curves taken with a scanning tunneling microscope on a synthetic, boron-doped diamond single crystal indicate that the diamond, boiled in acid and baked to $500^{\circ} \mathrm{C}$ in vacuum, does not exhibit ideal Schottky characteristics. These $I-V$ curves taken in ultrahigh vacuum do not fit the (raditional theory of thermionic emission; however, the deviation from ideal can be accounted for by charge screening at the diamond surface. At ambient pressure, the $I-V$ curves have a sharp threshold voltage at $1.7 \mathrm{eV}$ above the valence band edge indicating pinning of the Fermi energy. This measurement is in excellent agreement with the $\frac{1}{3}$ band gap rule of Mead and Spitzer [Phys. Rev. 134, A713 (1964)].
\end{abstract}

\section{INTRODUCTION}

Several group IV and III-V semiconductors have pinned Fermi levels that are predicted by the $\frac{1}{3}$ band gap rule of Mead and Spitzer. ${ }^{1}$ However, characterizing the electrical properties of diamond, a large band gap semiconductor $^{2}(5.5 \mathrm{eV})$ with high thermal conductivity ${ }^{3}$ $(\sim 20 \mathrm{~W} / \mathrm{cm} \mathrm{K})$ and a low dielectric constant ${ }^{4}(5.66)$ has been extremely difficult due to both the scarcity of natural conducting diamonds and the sparse information about their composition. Chemical vapor deposition of diamond thin films and synthesis of large, doped, high-quality diamonds ${ }^{5}$ by high pressure-high temperature techniques ${ }^{6}$ can provide samples with more manipulatable physical and electronic properties.

Current-voltage $(I-V)$ spectroscopy is often used to determine whether the nature of a metal-semiconductor contact is ohmic (having a linear response of the current to applied voltage) or Schottky (having a specific nonlinear response of the current to applied voltagc). Ideal Schottky $I-V$ curves demonstrate properties as described in detail by thermionic emission in metal-insulator-semiconductor (MIS) theory. ${ }^{7}$ Although other techniques can measure $I-V$ s at a given electrode separation, only the scanning tunneling microscope (STM) allows for a systematic examination of a variety of metal-diamond separations at the same site, thus insuring the same interface (gap) properties for each successive measurement. A set of $I-V$ 's are taken at a series of tip-sample separations defined by the set point current and sample bias. This separation is increased (decreased) between successive scans by increasing (decreasing) the sample bias. Although reports of imaging surfaces of thin diamond films with STM have been presented, ${ }^{8}$ no measurements of this type are systematically performed. Furthermore, the electrical characteristics of a thin, polycrystalline, or epitaxial diamond film may be different from those of a diamond crystal.

\footnotetext{
2) Present address: Chemistry Department, Harvey Mudd College, Claremont, California 91711.

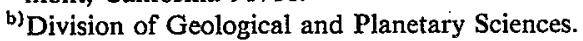

After unsuccessful attempts at imaging the surface of several natural type IIb diamonds and lightly doped, synthetic gem-quality diamonds with an STM, we obtained stable tunneling current and imaged ${ }^{9}$ a very highly borondoped, synthetic diamond. ${ }^{10}$ Surface composition of the large diamond used in the STM studies was determined by $\mathrm{x}$-ray photoelectric spectroscopy (XPS). Near-surface composition of similarly prepared diamonds was determined by ion milling/secondary ion mass spectroscopy (SIMS), since such analyses were not done on the large crystal at the request of the lender. The (111) face of the large diamond was confirmed by Laue diffraction and optical goniometry. A series of $I-V$ curves was taken on the diamond sample both at ultrahigh vacuum (UHV) and atmospheric pressure with two different tip compositions in order to examine the electrical properties of this diamond sample. The resulting curves are presented and discussed with regard to the theoretical calculations of Schottky emission.

\section{COMPOSITION OF SYNTHETIC DIAMOND}

SIMS measurements ${ }^{11}$ were performed on smaller, similarly synthesized GE boron-doped diamonds to determine elemental contaminants present in these samples. Analyses showed that the top $10 \mu \mathrm{m}$ were compositionally heterogeneous, with oxygen and hydrogen being the most prevalent contaminants. Boron (3-1500 ppm) and aluminum (30-400 ppm) were present as well as varying concentrations ( $<5000 \mathrm{ppm}$ ) of $\mathrm{Na}, \mathrm{Mg}, \mathrm{Si}, \mathrm{Cl}, \mathrm{K}, \mathrm{Ca}, \mathrm{Ti}, \mathrm{Fe}$, $\mathrm{Co}, \mathrm{Ni}, \mathrm{Cu}, \mathrm{Zr}, \mathrm{Ba}, \mathrm{W}$, and $\mathrm{Pt}$. Oxidized metallic catalyst inclusions are probably the source of many of these contaminants. Although aluminum has been previously considered to be a $p$-type dopant, careful analyses by Collins and Williams ${ }^{12}$ have shown aluminum to be electrically and optically inactive, despite its apparent role as a compensating "getter" for nitrogen. This idea is supported by the theoretical work of Eremets. ${ }^{4}$

The surface composition of the diamond used in the STM studies was determined from XPS using the $\mathrm{AlK}_{\alpha}$ emission at $1486 \mathrm{eV}$. A gold foil was used to make contact 
with the surface, and no surface charging was observed as noted by the binding energy of the $\mathrm{C} 1 s$ peak at $284.4 \mathrm{eV}$. Most natural diamonds show a charging ${ }^{13}$ of up to $6 \mathrm{eV}$. A surface illuminated spot size of 300 or $600 \mu \mathrm{m}$ in diameter was used.

Oxygen was the major surface contaminant observed by XPS, ${ }^{14}$ in addition to lesser amounts of $\mathrm{F}, \mathrm{Cl}, \mathrm{N}, \mathrm{Na}, \mathrm{Si}$, and Al. Oxygen (12\%) was observed after boiling the diamond in $\mathrm{HClO}_{4}$ and was reduced to $7 \%$ after $\mathrm{HF}$ etch in a glove box. This concentration was further reduced to $1.5 \%$ after $\mathrm{Ar}^{+}$sputter at $4500 \mathrm{eV}$. However, the location of the $\mathrm{C} 1 s$ peaks shifted and broadened suggesting that an amorphous or graphitized carbon had been formed during the sputtering. ${ }^{13}$ The transformation of a clean diamond surface to a graphite layer by argon ion etching at appropriate energies has been observed previously ${ }^{15}$ by Auger electron spectroscopy (AES) and is consistent with these results.

In a separate, subsequent XPS experiment, oxygen was persistent $(10 \%)$ after exposing the diamond to atmosphere and boiling in a non-oxygen-containing acid (HF). Further rinsing with $\mathrm{HCl}$ lowered the oxygen content slightly $(9 \%)$. Since oxygen was observed in large but varying quantities on the as-grown diamonds examined by SIMS, the observation of reduced oxygen after sputtering the large diamond sample may be indicative only of its heterogeneity rather than an actual cleaning. It should be noted that oxygen was not detected by SIMS on one spot on one of the other diamonds. Pate ${ }^{16}$ has observed by AES, which is not sensitive to hydrogen, that oxygen is the only measured surface contaminant on the polished, natural type II diamond surface, even after sample heating to greater than $500^{\circ} \mathrm{C}$ in ultrahigh vacuum. Photoemission spectroscopy (PES) measurements suggest that the surface is hydrogen terminated. The diamond surface is presumably highly inert unless extreme oxidation conditions are used, but the resident oxide is apparently very difficult to remove.

The effect of oxygen on the diamond surface is of great interest, especially in the attempt to preserve ohmic metaldiamond contacts. Mori et al. ${ }^{17}$ have demonstrated that an oxide layer changes previously ohmic contacts to Schottkylike contacts and that the Schottky dependence on the metal electronegativity disappears. They suggest that the oxide pins the Fermi level, thus changing the electrical properties of the metal-diamond interface.

\section{SURFACE POTENTIAL SCREENING AND REDUCED BAND-BENDING}

The large, synthetic diamond was boiled in $\mathrm{HNO}_{3}$, $\mathrm{H}_{2} \mathrm{SO}_{4}$, or $\mathrm{HF}$ and kept under ethanol until admitted into the STM chamber under nitrogen flow. The diamond was subsequently heated to temperatures up to $\sim 500^{\circ} \mathrm{C}$ in UHV. Contacts made to the surface with colloidal graphite or silver paint ${ }^{18,19}$ were verified to be ohmic by a two spot contact measurement. Indium contacts were shown to have a resistance much less than those used in the STM imaging

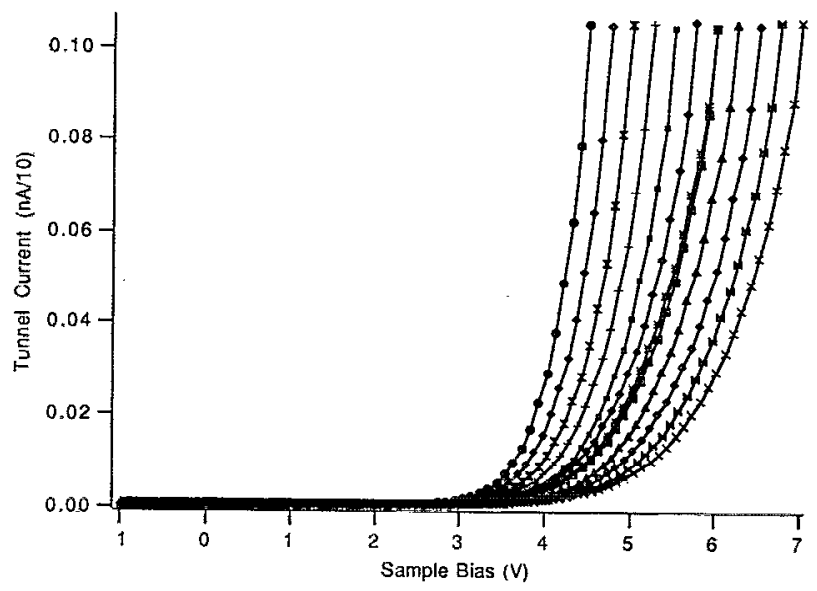

FIG. 1. A series of $I-V$ curves at different tip-sample separations on boron-doped diamond in $10^{-10}$ Torr. The feedback current was $1 \mathrm{nA}$ and original setpoint biases were $7.00,6.75,6.50,6.25,6.00,5.75,5.50,5.25$, $5.00,4.75$, and $4.50 \mathrm{~V}$, respectively, from right to left curves (from farther from the surface to closer). The tip was tungsten and electrical contact was made to the stub and the top of the diamond with indium.

experiments. Spectroscopic scans were taken at UHV and at ambient pressure. Cut platinum wire or etched tungsten wire was used as tips as indicated.

When reproducible spectroscopic curves were obtained, we observed no current for negative sample bias on any of the $I-V$ curves taken on the original $p$-type diamond at UHV or ambient using both the tungsten and platinum tips. However, the detailed nature of the curves obtained for the diamond in UHV was much different than that observcd in air. Shown in Fig. 1 are a series of $I-V$ curves taken at $1 \mathrm{nA}$ in UHV (low $10^{-10}$ Torr) using a tungsten tip at different tip-sample separations. As the separation increases, the rate at which the current rises decreases for each successive scan, as would be expected. The same results were observed on separate occasions at 100 pA feedback with a tungsten tip. Thus, for one order of magnitude range of current and $a \pm 7 \mathrm{~V}$ range of sample biases, the general nature of the $I-V$ spectroscopy appears similar.

The magnitude of the current depends on two factors: (1) the tunnel transmission probability, which in this discussion is assumed to be dependent primarily on the tip sample separation and bias independent and (2) the thermionic emission, which is proportional to the term $\left[\exp \left(-q V_{d} / k T-1\right]\right.$, where $q$ is the charge, $k$ is Boltzmann's constant, $T$ is the temperature, and $V_{d}$ is the surface voltage potential in the semiconductor which can be measured as the difference in potential between the conduction band edge at the surface and the bulk. $V_{d}$ is thus an indication of the extent of band bending.

The exponential character of the $I-V$ curves can be examined by a plot of $\ln (I)$ vs sample voltage as shown in Fig. 2 for the data of Fig. 1. An ideality factor or " $n$ factor" $(n)$ for a Schottky barrier can be calculated as explained by Card and Rhoderick. ${ }^{20}$ For sample biases greater than $3 k T / q, n$ reduces to 


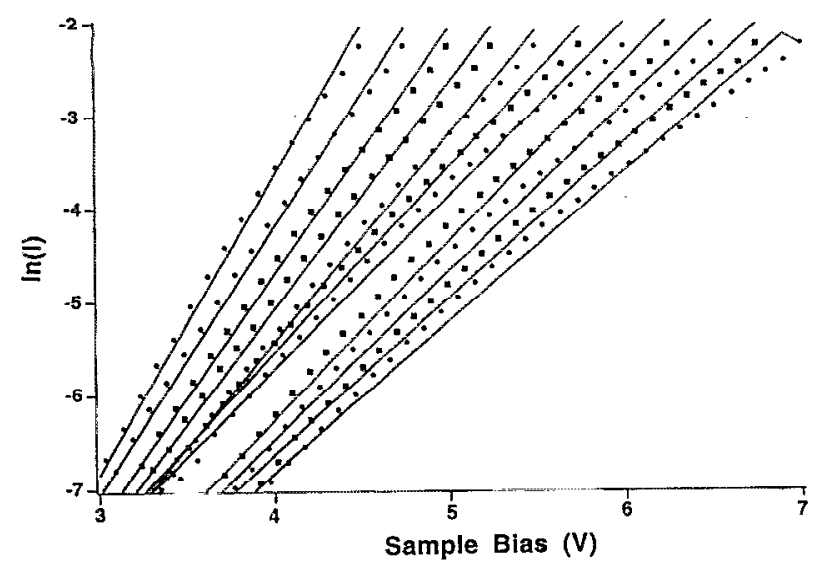

FIG. 2. Graph of natural $\log$ of the current vs sample bias for the data of Fig. 1, shown by the points. Also included are finear fits to the data, shown as solid lines.

$$
\frac{1}{n}=\frac{k T}{q} \times \frac{d \ln (I)}{d V}
$$

The calculated $n$ values from the data of Fig. 2 range from 6 to 12 for the data taken from a lower set point voltage (closer to surface) to higher voltage (farther from surface).

The $I-V$ curves cannot be explained by an ideal Schottky barrier $(n=1)$. The observed ratio of current change per volt is less than would be expected for the voltages applied when operating at physical separations conducive to tunneling. This result implies that the semiconductor is experiencing less applied voltage than would be expected for the bias applied to the tip. A mechanism for reducing the field at the semiconductor surface can be produced if the potential were screened by surface states on the semiconductor surface or by localized traps in the gap.

Based on the calculations of Cowley and Sze, ${ }^{21}$ Card and Rhoderick extend their analysis of the ideality factor to include the effect of surface states on the $I-V$ characteristics for a metal-insulator-semiconductor junction. For a given tip-sample separation $(s)$, depletion width ( $W$ $\left.=\sqrt{2 \epsilon_{s} \epsilon_{0} V_{d} / q N_{D}}\right)$, dopant concentration $\left(N_{D}\right)$, semiconductor dielectric constant $\left(\epsilon_{s}\right)$, density of surface statcs $\left(D_{S C}\right)$, and the vacuum permittivity $\left(\epsilon_{0}=8.854\right.$ $\times 10^{-12} \mathrm{~F} / \mathrm{cm}$ ), then the ideality factor can be expressed as

$$
n=1+\frac{s}{\epsilon_{0}}\left(\frac{\epsilon_{s}}{W}+q D_{S C}\right)
$$

Using average values for $V_{d}$, doping levels from the SIMS analysis $\left(10^{17}-10^{20} \mathrm{~cm}^{-3}\right)$, and diamond's dielectric constant, the surface state density was calculated to determine if reasonable physical values emerged (Fig. 3 ). The surface charge density calculated does fall within a reasonable range of values $\left(10^{13}-10^{14} \mathrm{~V}^{-1} \mathrm{~cm}^{-2}\right)$ and appears to depend primarily on the separation $(s)$. As is apparent from the graph in Fig. 3, the depletion width has much less effect on the calculated surface charge density than does the tipsample separation. However, since the depletion width de-

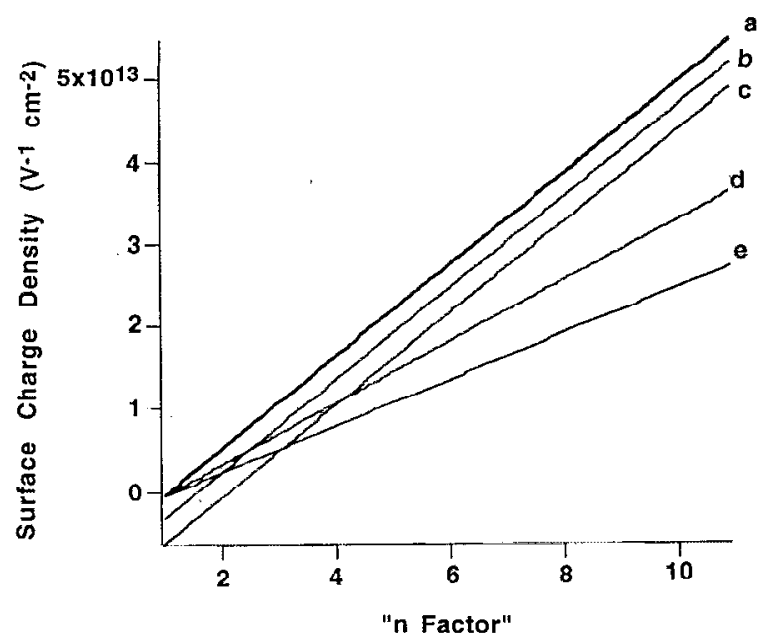

FIG. 3. Calculated surface charge density required to give the measured " $n$ factors" observed in the data of Fig. 2 . The plots correspond to constant values of the depletion width $(W)$ and separation $(s)$, both in $\AA$ identified as $(W, s)$ for: (a) $(W \geqslant 1000,10)$, (b) $(100,10)$, (c) $(50,10)$, (d) 1000,15$)$, (e) $(1000,25)$.

pends on the tip-sample separation and doping level, both of which are not known in the STM experiment, the graph was plotted for a large range of values. Interestingly, for the range of $n$ factors observed, the resultant $D_{S C}$ value is reasonable.

Geis et al. ${ }^{22}$ calculated an ideality factor of 2 and a barrier height of $1.3 \mathrm{~V}$ from $I-V$ measurements on a synthetic diamond at $580^{\circ} \mathrm{C}$ where most of the acceptors are ionized. They suggest that deep traps in the diamond are responsible for hysteresis in $I-V$ curves with tungsten contacts. No hysteresis was observed in our spectroscopic studies.

The slight curvature in the $\ln (I)$ vs $V$ plots of Fig. 2 is not unexpected. The assumptions used in this calculation are that the tunneling probability is constant over the range of biases used for each separation, $s$, and that the contribution to the current of minority carriers (electrons) to the forward bias current is minimal. The latter assumption is likely assured by the diamond's properties. The first assumption is not entirely valid in this system; the surface voltage potential produces band bending in the semiconductor that does have a nonlinear voltage and distance dependence becoming greater at larger separations. ${ }^{23}$ This band bending can be expressed as

$$
V_{d}=V_{d 0}\left\{\left[(c+1)^{2}-V / V_{d 0}\right]^{1 / 2}-c\right\}^{2},
$$

where $c$ is defined as $\left(\epsilon_{s} s / W\right)$, and $W_{0}$ and $V_{d 0}$ are the depletion width and surface potential as described before, but in the limit of zero separation. As observed in Fig. 2, the curvature increases at larger $s$ values (from left to right), consistent with the tunneling theory for finite separation.

\section{PINNING OF THE FERMI LEVEL}

The $I-V$ curves for the diamond surface in air show no such exponential dependence but display a strong thresh- 


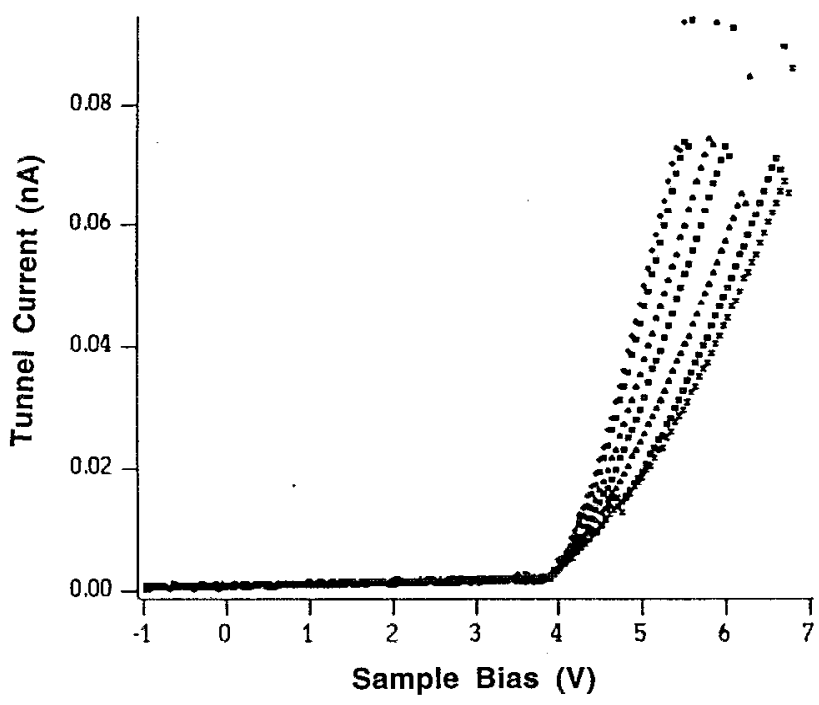

FIG. 4. A series of $I-V$ curves at different tip-sample separations established by different sample bias setpoints. $I-V$ 's were taken with a cut platinum tip at atmospheric pressure in air. Electrical contact was made to the stub with Ag paint. Original feedback voltages are, from left to right, $5.5,5.6,5.9,6.1,6.3,6.7$, and $6.8 \mathrm{~V}$.

old voltage and a nearly linear increase in current afterwards. Figure 4 shows a series of $I-V$ curves at an initial feedback current set to $100 \mathrm{pA}$ for a range from 4.0 to 6.0 $\mathrm{V}$. Similar curves were observed for the range of 7.0-8.0 V. The data were taken in air with a platinum tip and elecIrical contact to the stub with Ag paste. No current is observed below a consistent threshold at $+3.8 \mathrm{~V}$ sample bias for each separation. For all of the $I-V$ data collected in air, the scans were entirely reproducible at each site but variable between sites. None of the $I-V$ 's were exponential, but most had constant threshold voltages greater than $\sim+3.8 \mathrm{~V}$.

Because the diamond was exposed to the highly oxidizing conditions of hot perchloric acid before spectroscopic analysis, the presence of an oxide on the surface is likely despite the stability of the diamond surface to oxidation at ambient conditions. The XPS results verify the presence of tenacious oxygen after such a treatment. $\mathrm{Al}$ though the oxidation of the surface may not be complete, the small area probed by the STM tip may be sufficiently oxidized to change the electrical properties.

The threshold voltage seen in the $I-V$ curves of Fig. 4 does not change upon changing the bias set point of the tip (thus changing the tip-sample scparation for a constant current set point), suggesting that the Fermi level is pinned and that the band bending is small. The minimal band bending is consistent with the lack of exponential dependence of current on the voltage as would be expected for thermionic emission of a Schottky barrier. Instead, the threshold voltage is consistently measured at $3.8 \mathrm{~V}$ positive sample bias. With diamond's $5.5 \mathrm{eV}$ band gap, the Fermi level is thus observed to be pinned at $1.7 \mathrm{eV}$ above the valence band.

Studies using other techniques on type IIb diamonds have suggested the location of the pinned Fermi level.
Glover $^{24}$ made Schottky contacts on a GE boron-doped diamond by sputtering gold on the surface after cleaning the diamond by boiling in $\mathrm{HNO}_{3}$ and $\mathrm{H}_{2} \mathrm{SO}_{4}$. A zero-bias Fermi level is measured to be $1.73 \mathrm{eV}$ above the valence band maximum (the reference point for all future discussions), which agrees with the $\frac{1}{3}$ band gap rule of Mead and Spitzer. ${ }^{1}$ On natural type IIb diamonds, Mead and McGill $^{25}$ measured Fermi levels at $1.7-2.0 \mathrm{eV}$, while Himpsel et al. ${ }^{26}$ measured $1.3 \mathrm{eV}$. Certainly, the measured Fermi energy can be very different on differently prepared surfaces, while pinning is typically material related. The similarity between Glover's diamond, preparation, and results with those in this STM study suggest the same Fermi level pinning.

For the partially screened surface observed in vacuum, rectification is expected because, although the reverse bias is increased, the tip Fermi level does not catch up to the bending band edges. However, for a pinned Fermi level, one might expect filled states in the sample valence band to be observed at negative sample biases over $1.7 \mathrm{eV}$. However, the complete rectification is not surprising if one considers a simple model of basic tunneling theory, especially for the large band gap diamond.

The probability that an electron will tunnel based on the WKB approximation for a smoothly varying potential, when reduced to a simple square barrier, can be expressed $^{27}$ by the barrier penetration factor $D$,

$$
D=\exp (-\alpha \sqrt{\phi-e V} s)
$$

where $\alpha \sim 1 \mathrm{~V}^{-1 / 2} \AA^{-1}, e$ is the charge of the electron, $V$ is the voltage applied to the sample, and $\phi$ is the average work function of the tip and sample. For positive sample bias, the electrons at or near the Fermi energy of the tip tunnel to unfilled states in the sample is shown Fig. 5(a). As the bias is increased positively, these same electron levels close to the Fermi energy probe higher energy states on the sample. The electrons with energies much less than the Fermi energy will contribute to the current, but with an intensity exponentially less than the electrons having higher energies.

When the sample is biased negatively [Fig. 5(b)], the potential energy of the electrons in the sample are raised relative to those of the tip, and electrons will tunnel from filled states near the Fermi energy of the sample to the tip. As this bias is increased, the tunneling probability of the lower lying electrons decreases as they experience a tunnel barrier greater than the higher energy states. The contribution to the tunnel current from these lower lying electrons near the valence band edge decreases exponentially from the contribution of those states near the Fermi energy (three orders of magnitude).

Filled states, however, have been observed by STM on small band gap semiconductors such as silicon ${ }^{28}$ and gallium arsenide. ${ }^{29}$ One explanation asserts that these surface states may extend into the gap, and although the height of the barrier (energy) seen by the lower energy filled state electrons has increased, the barrier width decreases. The rectification observed on diamond is likely a result of the magnitude of diamond's band gap relative to these other 

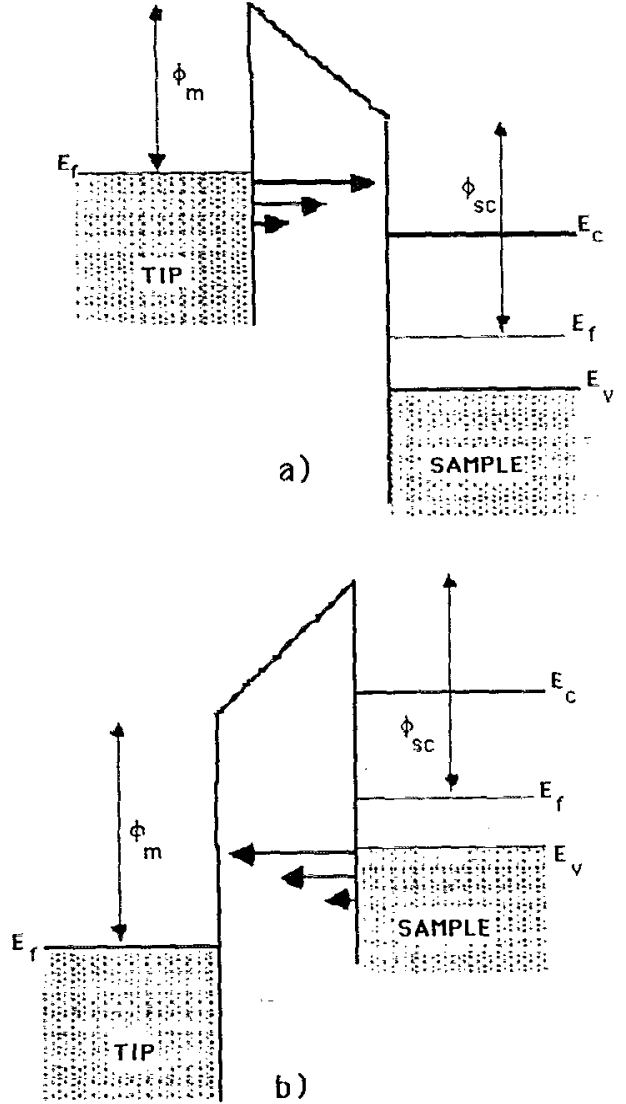

FIG. 5. Comparison of tunneling probabilities to examine unfilled states (top) and filled states (bottom) of a semiconductor sample with a metal tip. The inability to sce filled sample states due to the increased barrier felt by the electron at lower energy is discussed in the text. $\phi_{m}$ and $\phi_{s c}$ are the metal and semiconductor work functions. $E_{c}, E_{v}$, and $E_{f}$ are the conduction and valence bands and the Fermi energy, respectively.

semiconductors. The probability of valence band electrons tunneling through the barrier presented by a $5.5 \mathrm{eV}$ gap at tip-sample distances attained for these STM measurements (due to high sample bias and low tunnel current) is very small.

\section{CONCLUSION}

The presence of persistent oxygen on the surface of a synthetic, boron-doped, single crystal diamond is thought to account for the charge screening observed in vacuum spectroscopy. The unpinned character is reproducible, and calculations suggest that partial screening of the applied bias is responsible for the deviations from ideal Schottky character. The threshold voltage observed in air is consistent with pinning the Fermi level at $\sim 1.7 \mathrm{eV}$. Surface conduction and metal impurities close to the surface may also have a considerable effect on the nature of the localized electrical properties.

Due to the heterogeneity of the diamond, different localized areas on the surface in vacuum have slightly different properties. Some areas were insulating, as indicated by the inability to draw significant current for given feedback conditions on numerous occasions. ${ }^{9}$ However, currentvoltage spectroscopy not only conforms to the $\frac{1}{3}$ band gap rule observed for other types IV and III-V semiconductors, but also confirms the need for high voltage biases for successful STM imaging operation.

\section{ACKNOWLEDGMENTS}

This work was supported by the NIH National Research Science Award and the Office of Naval Research. SIMS analyses were generously performed by Dr. Robert G. Wilson of Hughes Research Laboratories. XPS work was done with Dr. Richard Vasquez at the Jet Propulsion Laboratory. S. Baker thanks Dr. William Kaiser of the Jet Propulsion Laboratory for valuable discussions, and General Electric, Schenectady, NY, for use of their synthetic diamonds.

${ }^{1}$ C. A. Mead and W. G. Spitzer, Phys. Rev. 134, A713 (1964).

${ }^{2}$ F. C. Champion, Electronic Properties of Diamond (Butterworth, London, 1983), p. 25.

${ }^{3}$ CRC Handbook of Chemistry and Physics, edited by R. C. Weast and M. J. Astle (CRC, Boca Raton, 1981), p. E-11.

${ }^{4}$ M. I. Eremets, Semicon. Sci. Technol, 6, 439 (1991).

${ }^{5}$ H. M. Strong, Am. J. Phys. 57, 794 (1989); H. M. Strong and R. E. Hanneman, J. Chem. Phys. 46, 3668 (1967).

${ }^{6}$ F. P. Bundy, H. T. Hall, H. M. Strong, and R. H. Wentorf, Nature 4471, 51 (1955); H. P. Bovenkerk, F. P. Bundy, H. T. Hall, H. M. Strong, and R. H. Etorf, Jr., Nature 184, 1094 (1959).

${ }^{7}$ S. M. Sze, Semiconductor Devices: Physics and Technology (Wiley, New York, 1985).

${ }^{8}$ For example, see T. Tsuno, T. Imai, Y. Nishibayashi, K. Hamada, and N. Fuminori, Jpn. Appl. Phys. 30, 1063 (1991); H. G. Busmann, H. Sprand, I. V. Hertel, W. Zimmernann-Edling, and H. J. Guntherodt, Appl. Phys. Lett. 59, 295 (1991).

${ }^{9}$ S. M. Baker, G. R. Rossman, and J. D. Baldeschwieler, J. Diamond Related Mater. (to be published).

${ }^{10}$ Prepared by General Electric, Schenectady, NY, by a high pressure, high temperature technique as in Ref. 5 .

"R. G. Wilson and S. W. Novak, J. Appl. Phys. 69, 466 (1991).

${ }^{12}$ A. T. Collins and A. W. S. Williams, J. Phys. C. 4, 1789 (1971).

${ }^{13}$ F. R. McFeely, S. P. Kowalczyk, L. Ley, R. G. Cavell, R. A. Pollak, and D. A. Shirley, Phys. Rev. B 9, 5268 (1974).

${ }^{14}$ The XPS studies are insensitive to the small concentrations of many of the elements (including the boron dopant) found with SIMS and are unable to detect hydrogen.

is A. Hoffman, P. J. K. Paterson, and S. Prawer, Nucl. Instrum. Methods Phys. Res. B 52, 63 (1990).

${ }^{16}$ Brad. B. Pate, Surf. Sci. 165, 83. (1986)

${ }^{17}$ Y. Mori, H. Kawarada, and A. Hiraki, Appl. Phys. Lett. 58, 940 (1990).

${ }^{18}$ B. Pate (private communication, June 1991); Y. Mori, H. Kawarada, and A. Hiraki, Appl. Phys. Lett. 58, 940 (1990).

${ }^{19}$ K. L. Moazed, J. R. Zeidler, and M. J. Taylor, J. Appl. Phys. 68, 2246 (1990).

${ }^{20}$ H. C. Card and E. H. Rhoderick, J. Phys. D. 4, 1589 (1971).

${ }^{21}$ A. M. Cowley and S. M. Sze, J. Appl. Phys. 36, 3212 (1965).

${ }^{22}$ M. W. Geis, D. D. Rathman, D. J. Ehrilich, R. A. Murphy, and W. T. Lindley, IEEE Electron. Dev. Lett. EDL-8, 341 (1987).

${ }^{23}$ L. D. Bell, W. J. Kaiser, M. H. Hecht, and F. J. Grunthaner, Appl. Phys. Lett. 52, 278 (1988).

${ }^{24}$ G. H. Glover, Solid-State Electron. 16, 973 (1973).

${ }^{25}$ C. A. Mead and T. C. McGill, Phys. Lett. 58A, 249 (1986).

${ }^{26}$ F. J. Himpsel, P. Heinemann, and D. E. Eastman, Solid State Commun. 36, $631(1980)$.

${ }^{27}$ C. B. Duke, Tunneling in Solids (Academic, New York, 1969), p. 61.

${ }^{28}$ R. Wolkow and Ph. Avouris, Phys. Rev. Lett. 60, 1049 (1988).

${ }^{29}$ P. Mărtensson and R. M. Feenstra, Phys. Rev. B 39, 7744 (1989). 